\title{
Competencias de innovación en entornos virtuales de aprendizaje basados en gestión del conocimiento
}

Innovation Competences in Virtual Learning Environments based on Knowledge Management

Competências de inovação em ambientes virtuais de aprendizagem baseado na gestão do conhecimento

\section{Francisco Xavier Ampuero Velásquez}

Escuela Politécnica Nacional (Quito, Ecuador)

fampuero@uazuay.edu.ec

https://orcid.org/0000-0003-3269-5049

\author{
Valentina Ramos Ramos \\ Escuela Politécnica Nacional (Quito, Ecuador) \\ valentina.ramos@epn.edu.ec \\ https://orcid.org/0000-0001-7803-8461 \\ Francisco Salgado Arteaga \\ Universidad del Azuay (Cuenca, Ecuador) \\ fsalgado@uazuay.edu.ec \\ https://orcid.org/0000-0002-9253-1276
}

DOI: https://doi.org/10.32719/25506641.2020.7.9

Presentado: 30 de agosto de 2019 • Revisado: 21 de octubre de 2019

Aceptado: 28 de noviembre de 2019

Artículo de investigación

Licencia Creative Commons

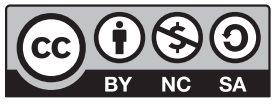




\section{Resumen}

El presente artículo muestra el estado de situación de las teorías de gestión del conocimiento, incluyendo la tipología del aprendizaje organizacional y los estilos personales. Estas teorías, sobre todo las relacionadas con los diferentes estilos de aprendizaje personal, deben ser consideradas en la creación de programas de e-learning en entornos de aprendizaje virtual (EVA). De esta forma, dichos programas pueden propender al desarrollo de competencias de innovación en los participantes. El objetivo del artículo fue definir escenarios por cada estilo de aprendizaje, los cuales sirven de guía para individuos que educan y lideran equipos de trabajo. Se realizó una investigación cualitativa con base en fuentes secundarias y revisión de literatura. Entre los principales hallazgos, se resalta la importancia de contar con equipos de estudio o trabajo que contengan, de ser posible, individuos con todos o la mayoría de estilos de aprendizaje personal, que puedan pasar por las diferentes etapas del proceso de innovación sin estancarse en el aislamiento académico abstracto, donde nos quedamos solo en ideas y conceptos, ni el otro extremo, con aplicaciones prácticas muy simples que se hacen sin reflexión previa.

Palabras clave: Gestión del conocimiento, e-learning, entornos virtuales de aprendizaje, competencias de innovación, estilos de aprendizaje.

JEL: O31 Innovación e invención: procesos e incentivos; O32 Gestión de innovación tecnológica en instituciones de educación e investigación.

\section{Abstract}

This article shows the status of knowledge management theories, including the typology of organizational learning and personal styles. These theories, especially those related to the different styles of personal learning should be considered in the creation of E-Learning programs in Virtual Learning Environments (VLE). In this way, such programs can tend to the development of innovation competencies in participants. The objective of the article was to define scenarios for each learning style, which serve as a guide for individuals who educate and lead work teams. Qualitative research was conducted based on secondary sources and literature review. Among the main findings, it is highlighted the importance of having study or work teams that have, if possible, individuals with all or most of the personal learning styles, and that they can go through the different stages of the innovation process without staying in the abstract academic isolation where we remain only in ideas and concepts, or the other extreme, with very simple practical applications that are made without prior reflection.

Keywords: Knowledge Management, E-Learning, Virtual Learning Environments, Innovation Competencies, Learning Styles.

JEL: O31: Innovation and invention: processes and incentives; O32 Technological innovation management in education and research institutions.

\section{Resumo}

O presente artigo mostra o estado de situação das teorias de gestão do conhecimento, incluindo a tipologia da aprendizagem organizacional e os estilos pessoais. Estas teo- 
rias, principalmente as que estão relacionadas com os diferentes estilos de aprendizagem pessoal, devem ser consideradas na criação de programas de E-Learning em Entornos de Aprendizagem Virtual (EAV). Desta forma, ditos programas podem promover o desenvolvimento de competências de inovação nos participantes. O objetivo do artigo foi definir cenários por cada estilo de aprendizagem, os quais servem de guia para indivíduos que educam e lideram equipes de trabalho. Realizou-se uma pesquisa qualitativa com base em fontes secundárias e revisão de literatura. Entre as principais descobertas, ressalta-se a importância de contar com equipes de estudo ou trabalho que tenham, caso seja possível, indivíduos com todos ou a maioria dos estilos de aprendizagem pessoal, e que estes possam passar por diferentes etapas do processo de inovação sem permanecerem nem no isolamento acadêmico abstrato, onde ficam somente em ideias e conceitos, nem no outro extremo, com aplicações práticas muito simples e feitas sem reflexão prévia.

Palvras-chave: Gestão do conhecimento, e-learning, ambientes virtuais de aprendizagem, competências de inovação, estilos de aprendizagem

JEL: O31 Inovação e invenção: processos e incentivos; O32 Gestão da inovação tecnológica em instituições de ensino e pesquisa.

\section{Introducción}

E xiste una relación metabólica y simbiótica entre la gestión del conocimiento y el aprendizaje organizacional a través del e-learning en entornos virtuales de aprendizaje (EVA), donde las personas tienen formas específicas de aprender según sus preferencias y experiencias personales. Estos estilos fueron analizados por el teórico educativo norteamericano David A. Kolb desde los años 60 para desarrollar sus teorías sobre el aprendizaje experimental. La educación virtual, basada en la gestión del conocimiento y los estilos de aprendizaje, permite educar personas en diferentes organizaciones de forma casi omnipresente: en cualquier parte y a cualquier hora. Al mismo tiempo, las nuevas herramientas tecnológicas permiten mejorar la generación y transformación del conocimiento tácito en explícito, así como su almacenamiento y difusión.

La enseñanza-aprendizaje en línea, virtual o digital, también conocida como e-learning, se define como una modalidad formativa en la que se utilizan medios didácticos para aprender un contenido concreto en el marco de una institución; esta formación se realiza por medios electrónicos, ya que existe entre profesor y estudiante, o entre instructor y participante, una separación física y posiblemente temporal, aunque no necesariamente (Barberà 2008). 
Sobre este aspecto, y desde una perspectiva más específica, uno de los mayores retos que han enfrentado la educación y la capacitación en las últimas décadas tiene relación con la virtualización de los entornos de aprendizaje. Se ha tratado de convertir dichos entornos virtuales en espacios de enseñanza, toda vez que esta es parte del rol fundamental del docente o instructor empresarial, por lo que debe existir su compromiso para realizar dicha actividad. Asimismo, un compromiso por parte de quien recibe la formación es llegar a un aprehendizaje, un nivel superior de aprendizaje, no solo para el momento o corto plazo, sino para la vida; es decir, se trata de convertir en hábitos a los conocimientos adquiridos.

Esto lo explica el pedagogo Brito Albuja (2014), y agrega que los estudiantes de una carrera o los participantes de una capacitación deben ser capaces de hacer productivo el conocimiento. Por ello, los contenidos de los EVA, más que ser un listado de conocimientos específicos a ser transmitidos, deben propender a la generación de competencias interdisciplinarias, formativas y profesionales. De igual forma, estas deben estar ajustadas a las necesidades del mercado laboral y al contexto en que se desenvuelve el aprehendiz, a quien se le pueden transmitir dichos contenidos usando diferentes estrategias y en diversos lugares y tiempos.

El concepto competencias de innovación tiene mayor amplitud, en comparación con habilidades de innovación, ya que ser competente implica tener varias habilidades relacionadas a la innovación (Velayudhan y Maran 2010). En este caso, no solo se refiere a saber cómo generar ideas innovadoras, sino a estar en la capacidad de hacerlo en situaciones reales, problemáticas o de incertidumbre e, inclusive, a tener la actitud y la predisposición para llevarlas a la práctica. Estos aspectos le dan, al docente o instructor empresarial, el poder de transmitir la competencia de forma teórica y práctica (Bjornali y Støren 2012).

Para lograr el desarrollo de competencias, es necesaria la aplicación de teorías de la gestión del conocimiento, entendida como "el desarrollo de tecnologías, metodologías y estrategias para su medición, creación y difusión" (Rodríguez Gómez 2006, 25). Además, se debe visualizar a las empresas, industrias y universidades como organizaciones de aprendizaje dentro de la sociedad del conocimiento, que genera "una serie de procesos relacionados con la generación o captación de conocimiento, transformación, transferencia, almacenamiento y reutilización de este conocimiento" (Ramos 2017, 
11), que estimulan varios tipos de innovaciones: de producto (bien o servicio), de proceso, de mercadotecnia o comercialización, o de organización o prácticas internas (OCDE 2007).

A continuación, se amplían las teorías y los conceptos indicados en los párrafos anteriores, como marco de referencia para generadores de programas de e-learning en EVA. De esta forma, los docentes o instructores empresariales podrán considerar los estilos de aprendizaje individuales y utilizar los diferentes escenarios para promover el desarrollo de competencias en los procesos de innovación.

\section{Materiales y métodos}

La metodología empleada está basada en la investigación documental y bibliográfica de fuentes secundarias. La búsqueda de información se enfocó en las principales teorías de la gestión del conocimiento, aprendizaje organizacional mediante e-learning en EVA, e innovación centrada en la generación de competencias para su aparición en individuos inmersos en diferentes tipos de procesos formativos, con base en sus estilos de aprendizaje personal.

\section{Análisis y resultados}

\section{Gestión del conocimiento y aprendizaje organizacional}

Peter Drucker (1993) predijo, a finales del siglo pasado, que el conocimiento será el elemento asociado a la dimensión humana que se convertirá en el principal factor de producción. Esta predicción se ha vuelto realidad y en esto coinciden las empresas y la academia en el siglo XXI.

Entre las corrientes de pensamiento relacionadas con la gestión del conocimiento, se parte de la teoría social del aprendizaje de Wenger (2001), quien establece los componentes principales para que las personas aprendan en cualquier comunidad de práctica desde el ámbito personal, de trabajo o enseñanza, presencial o virtual: 
Figura 1

Comunidades de práctica

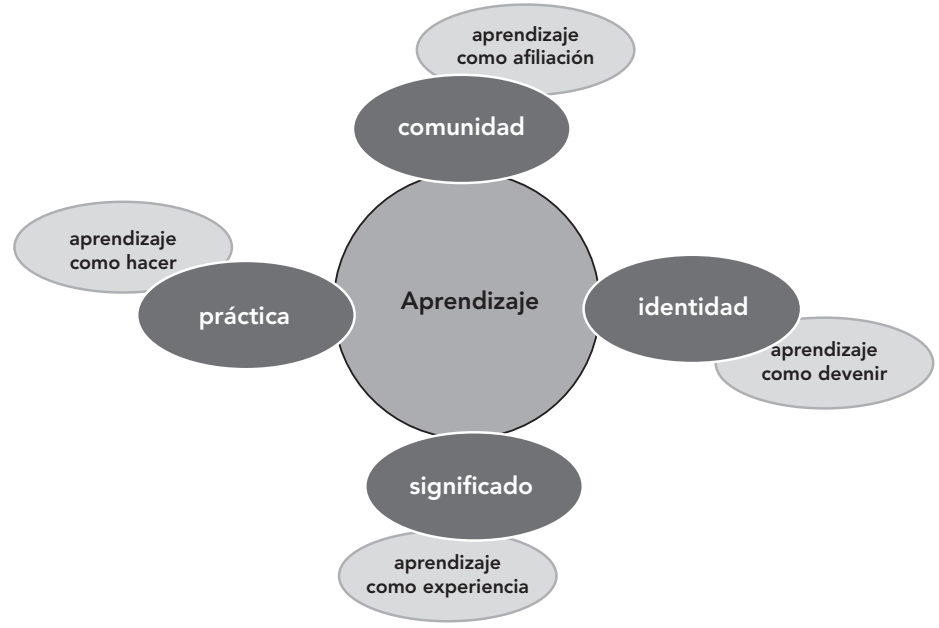

Fuente: adaptado de Wenger $(2001,23)$.

El significado se refiere al aprender como experiencia; la práctica, al aprender cómo hacer; la comunidad, al aprender como pertenencia, y la identidad, al aprender como devenir o llegar a ser.

Dicha teoría replantea el aprendizaje: al no ser una actividad separada, vinculada únicamente con programas educativos, los seres humanos aprenden en varias etapas de su vida; inclusive, a veces el aprendizaje se intensifica "cuando las situaciones hacen tambalear nuestro sentido de la familiaridad, cuando nos vemos desafiados más allá de nuestra capacidad de respuesta, cuando deseamos comprometernos con nuevas prácticas o intentamos unirnos a nuevas comunidades" (Wenger 2001, 25). Su marco teórico es muy fundamentado, ya que abarca teorías de estructura social, por un lado, y de la experiencia situada, por el otro. De igual forma, en un extremo utiliza las teorías de la práctica y en el otro, las de la identidad; en el intermedio, trabaja con teorías del poder, la colectividad, el significado y la subjetividad. Por este motivo, en los EVA se debe propender a que las personas aprendan de la forma en que han aprendido todas aquellas cosas que les han resultado útiles e interesantes en su vida. 
Figura 2

Teoría social del aprendizaje

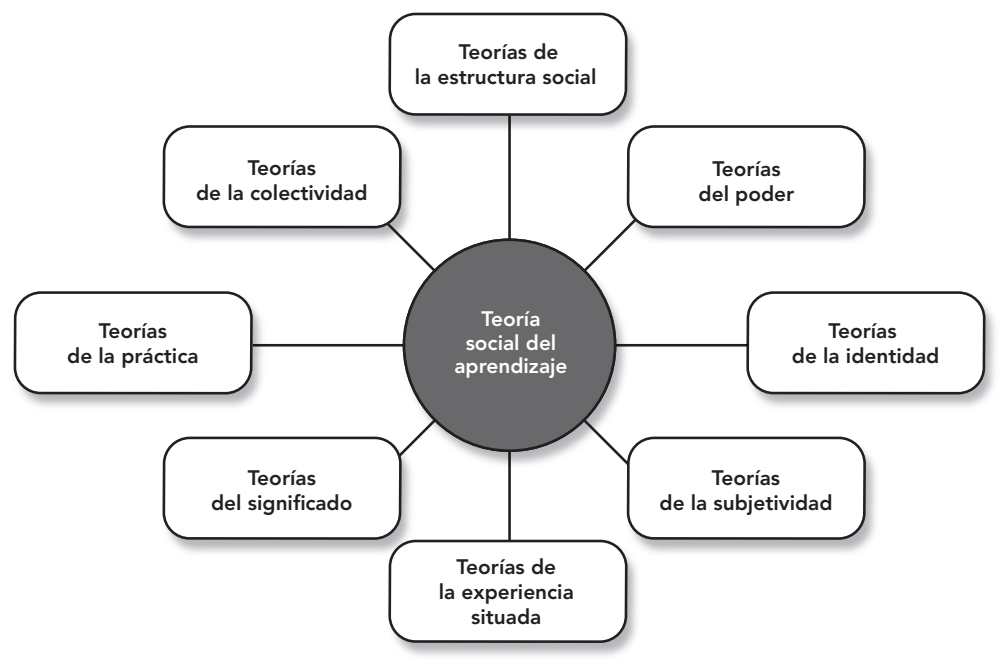

Fuente: adaptado de Wenger $(2001,34)$.

Desde la óptica académica y como ámbito geográfico, se estudia a la gestión del conocimiento desde dos perspectivas: la norteamericana, basada en la preocupación de las organizaciones por capitalizar el conocimiento para la toma de decisiones, y la oriental, sustentada en cada trabajador y sus procesos individuales, en los que tornan explícitos sus conocimientos tácitos. Sobre esta última visión, es necesario destacar la obra de Ikujiro Nonaka, quien, junto a varios colaboradores, ha redefinido a través de los años la gestión del conocimiento hacia una gestión basada en el conocimiento, no solo como herramienta sino como una teoría hacia un nuevo paradigma en la economía del conocimiento (Nonaka y Toyama 2006).

En su libro La organización creadora de conocimiento, Nonaka y Takeuchi (1999) buscan explicar cómo las compañías japonesas crearon la dinámica de la innovación en sus organizaciones, sobre todo en las décadas de los 70 y 80 . Además, presentan una teoría de creación de conocimiento desde su propia naturaleza, sus dos dimensiones, que pueden ser apreciadas en la siguiente figura. 
Figura 3

\section{Dimensiones de la creación del conocimiento}

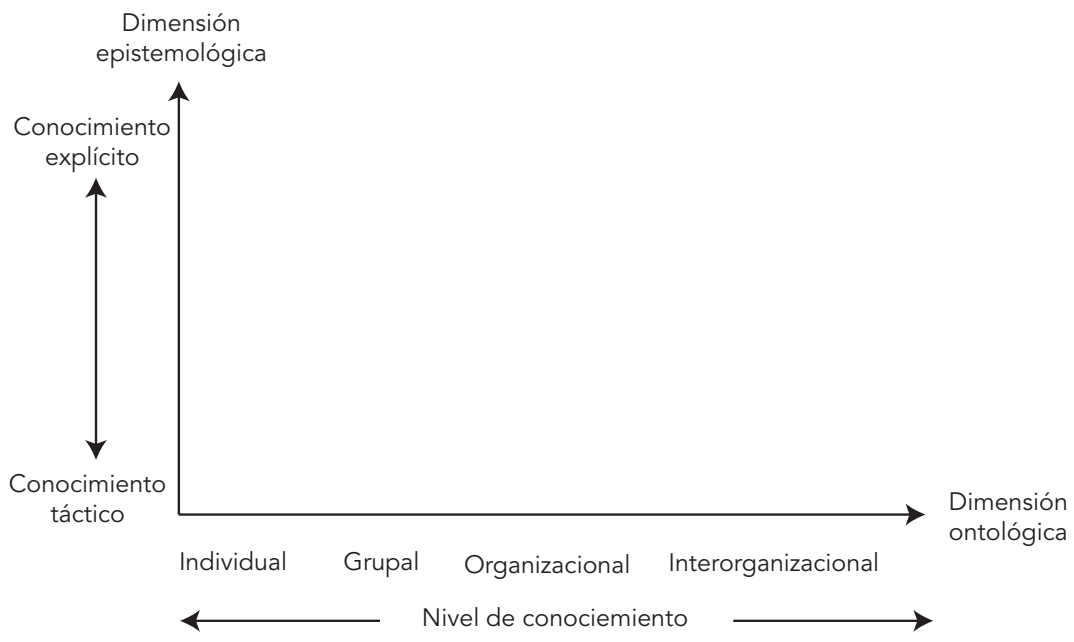

Fuente: adaptado de Nonaka y Takeuchi $(1999,62)$.

a) Dimensión ontológica: se centra en niveles de entidades creadoras individuales, grupales, organizacionales e interorganizacionales, es decir, basadas en el entorno y el impacto potencial de los flujos de conocimiento. Sin embargo, resalta al individuo como la base de creación de conocimiento; la organización debe apoyar su creatividad y proveer el ambiente contextual para este fin. Luego, debe respaldar, amplificar y cristalizar dicha generación de conocimiento.

b) Dimensión epistemológica: se basa en procesos de comunicación que convierten el conocimiento y su contenido en conocimiento tácito (personal, no formal, difícil de transmitir, con raíces en la experiencia individual más los valores, emociones, ideales, etc.) y en conocimiento explícito (expresable a través de lenguaje formal, con palabras, números, etc., por lo que es más factible de compartir con datos, fórmulas, procedimientos, códigos y principios universales; esto se puede almacenar en medios de soporte físico, impresos o digitales, o virtual).

Esta transformación se puede dar de cuatro formas diferentes, como se muestra en la siguiente figura. 
Figura 4

Cuatro formas de conversión de conocimiento y su contenido

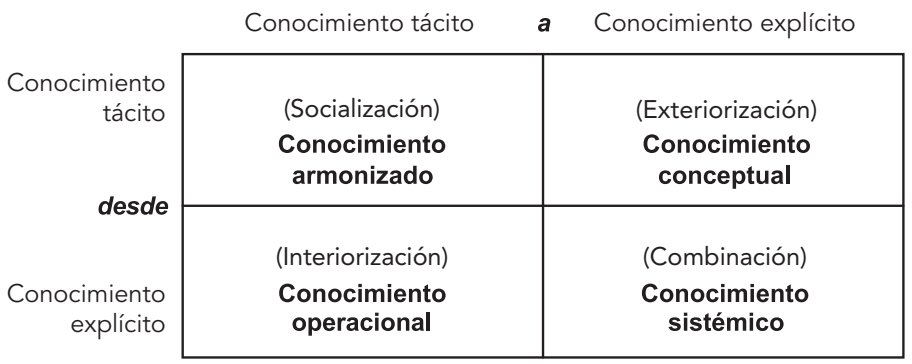

Fuente: adaptado de Nonaka y Takeuchi $(1999,80)$.

La socialización es el proceso de adquirir conocimiento tácito a través de la imitación y la práctica, al compartir experiencias de forma oral (exposiciones, clases, demostraciones, etc.) o escrita (libros, manuales, comunicaciones, etc.). Se inicia con la creación de un campo de interacción con este objetivo, y es un conocimiento armonizado.

La exteriorización, por su parte, es el proceso de conversión del conocimiento tácito en conceptos explícitos, para que otros miembros de la organización lo puedan comprender. Presupone la interacción entre individuo y grupo, y requiere técnicas para expresar dicho conocimiento con lenguaje figurativo, analogías, metáforas, lenguaje visual, etc. Se la llama conocimiento conceptual.

La interiorización es el proceso de incorporar conocimiento científico explícito a partir del tácito, con base en el concepto de aprender haciendo. A partir de la puesta en práctica del nuevo conocimiento, se analizan las experiencias adquiridas, que se vuelven base del conocimiento tácito de los miembros de la organización, en la forma de prácticas de trabajo o mapas mentales compartidos. Se conoce como conocimiento operacional.

La combinación, finalmente, es el proceso de sistematización de conceptos en conocimiento explícito, el cual se sintetiza y formaliza para que cualquiera en la empresa pueda acceder a él. Se requiere realizar la captura e integración del nuevo conocimiento explícito, difundirlo con conferencias, 
presentaciones, etc., y procesarlo para que se vuelva más accesible. Este el conocimiento sistémico.

A partir de estas formas y sus posibles combinaciones, se grafica un espiral de dentro hacia fuera, que se mueve a través de las dimensiones ontológicas y epistemológicas. Implica que la organización debe generar entre su personal "el contexto apropiado para facilitar las actividades grupales para la creación y acumulación de conocimiento a nivel individual" (Nonaka y Takeuchi 1999, 80). Al final, la espiral, al pasar por las formas de conversión del conocimiento, se visibiliza por cinco condiciones: intención (visión), autonomía (empoderamiento), fluctuación y caos creativo (reflexión en la acción), redundancia (diferentes puntos de vista) y variedad de requisitos (diversidad y complejidad interna), situación que genera la siguiente figura:

Figura 5

\section{La espiral del conocimiento}

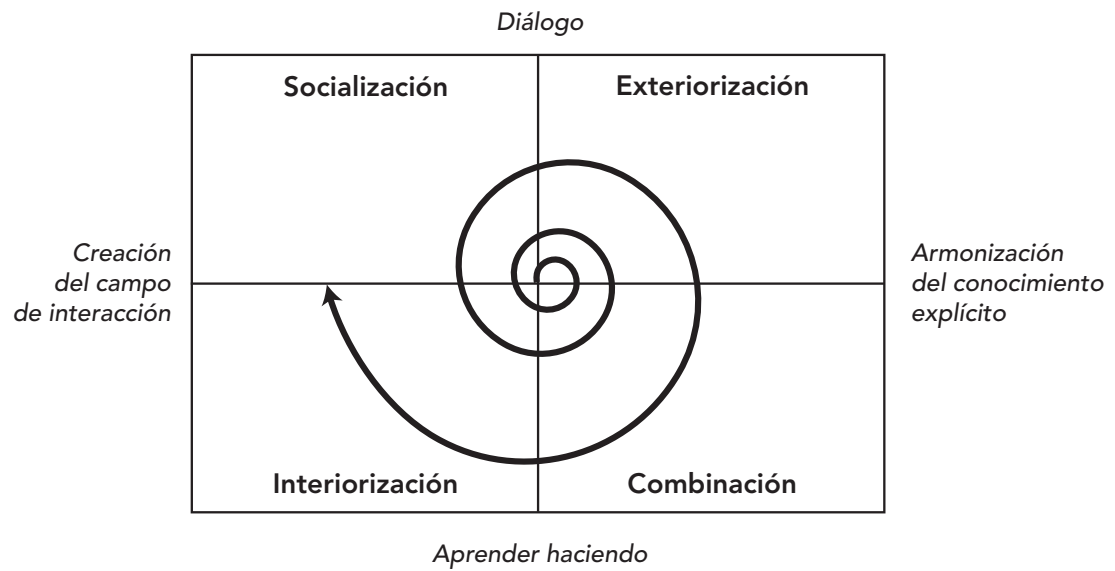

Fuente: adaptado de Nonaka y Takeuchi $(1999,81)$.

Desde la visión norteamericana, dos de los autores más prolíficos sobre esta temática, Davenport y Prusak (2001), escribieron un libro clásico con el título Conocimiento en acción: cómo las organizaciones manejan lo que saben. En él manifiestan que hubo un punto de inflexión en que la gestión del conocimiento pasó de ser una teoría académica a ser un componente esencial 
de la vida de las organizaciones; ese punto de inflexión estableció al conocimiento con un elemento primordial generado y usado por las organizaciones saludables. Estas interactúan con su entorno -absorbiendo información y transformándola en conocimiento- para luego realizar sus actividades combinando dicho conocimiento con sus experiencias, valores y normas internas. Se trata de un proceso metabólico donde "sienten y responden. Sin conocimiento, una organización no se podría organizar a sí misma" (Davenport y Prusak 2001, 61). Con estas premisas, acuñaron la siguiente definición: "La gestión del conocimiento se basa en los recursos existentes que la organización ya posee: buena gestión de sistemas de información, gestión del cambio organizacional y prácticas de gestión de recursos humanos" (Davenport y Prusak 2001, 163).

En relación con el conocimiento que genera el aprendizaje organizacional, Lundvall (1999) considera que "la estrategia más prometedora para el crecimiento económico está dirigida a fortalecer la base de conocimiento de la economía" (Lundvall 1999, 14). Hace hincapié en que la información y el conocimiento están en contextos diferentes, ya que el primero representa datos seleccionados para la toma de "decisiones racionales de los agentes individuales", mientras que el segundo puede considerarse un activo, ya sea un factor en el proceso productivo o, inclusive, una mercancía normal en la "línea de los productos tangibles reproducibles" (Lundvall 1999, 16).

Además, manifiesta que la mayoría del conocimiento no es completamente público ni tampoco completamente privado, ya que "la base del conocimiento está fragmentada y conformada por depósitos semipúblicos cuyo acceso está repartido de forma regional, profesionalmente y a través de redes" (Lundvall 1999, 18). Por último, lo clasifica en cuatro categorías (Lundvall y Johnson 1994):

a) Saber qué (know-what): conocimiento de los hechos, próximo a lo que se conoce como información conformada por datos específicos que pueden ser comunicables.

b) Saber por qué (know-why): conocimiento de principios y leyes de la naturaleza, la mente humana y la sociedad. Es de extrema importancia en el desarrollo tecnológico basado en la ciencia, pues permite avanzar más rápido y reducir errores en procesos de prueba y error. 
c) Saber cómo (know-how): cualificaciones o capacidades para hacer algo. Pueden ser las habilidades artesanales o de trabajadores de producción, pero actualmente son clave en toda actividad humana y económica. Aun la ciencia requiere habilidades de intuición.

d) Saber quién (know-who): de creciente importancia, implica información sobre quién sabe qué y/o quién sabe hacer qué. También representa la capacidad social para comunicarse y cooperar entre profesionales y expertos de varias disciplinas, e individuos en general.

Todas estas clasificaciones del conocimiento podrían ser generadas por el aprendizaje interactivo institucional para la generación de innovaciones planteado por Johnson (1992), entendido como la combinación de learning-by-searching -que corresponde a la investigación y representa una búsqueda permanente de alternativas a los problemas de innovación, con el fin de estimularla de la mejor manera-y de learning-by-exploring - un esquema generado por las actividades de investigación básica llevadas a cabo por redes sociotécnicas, académicas o similares dedicadas a este fin (Daza 2012)-. Estos dos elementos, orientados por la acción (action oriented) y el beneficio social, crean la base de un nuevo paradigma tecnológico dentro de una dimensión dinámica y radical de gran importancia en el largo plazo.

Por otra parte, el learning-by-producing es un subproducto de actividades económicas organizadas con otros fines primarios. Se lo considera un aprendizaje que se liga con actividades rutinarias de producción y distribución; por lo tanto, está orientado por la estructura (structure oriented). De esta modalidad parten el learning-by-doing -una de las principales teorías de Kenneth J. Arrow (1962), pionero de los estudios en que los operarios aumentaban su conocimiento al realizar su actividad productiva, con importantes implicaciones en la economía de empresas y países- y el learningby-using -ya que al usar sistemas complejos es posible incrementar su eficiencia, como sucede con los clientes o consumidores de un producto, cuya retroalimentación permite mejorarlo e innovarlo-.

Adicionalmente, el learning-by-interacting - presentado por Arrow (1962) y desarrollado por Lundvall (1992) como parte fundamental de los sistemas nacionales de innovación que vincula las teorías de innovación y el aprendizaje interactivo de Arrow- se basa en la interacción entre productores y usuarios, que genera innovaciones de producto. Su importancia radica en el 
carácter acumulativo del conocimiento que puede generarse en la organización, para luego traducirse en fortalecimiento de la capacidad tecnológica local. De este también se desprende el learning-from-network, el aprendizaje interorganizacional (Lane y Lubatkin 1998), que comprende el aprendizaje interactivo en el marco de las relaciones interorganizacionales y las redes de investigación. La fuente de ideas e innovación se puede captar fuera de las fronteras de la organización y absorber mediante el aprendizaje por el intercambio de nuevas informaciones, conocimientos y competencias interdisciplinarias, compartidas de manera formal o informal entre los distintos actores organizacionales de la red, para generar productos e innovaciones.

\section{Definición de innovación y competencias de innovación}

En su obra Teoría del desenvolvimiento económico, Joseph A. Schumpeter (1997) define a la innovación como la energía empresarial que genera una destrucción creadora y es la fuente de todo cambio en las estructuras económicas de los países, sobre todo en los sistemas capitalistas (Schumpeter 1997). El Manual de Oslo, dentro de sus definiciones básicas, establece que "una innovación es la introducción de un nuevo, o significativamente mejorado, producto (bien o servicio), de un proceso, de un nuevo método de comercialización o de un nuevo método organizativo, en las prácticas internas de la empresa, la organización del lugar de trabajo o las relaciones exteriores" (OCDE 2007, 56).

Desde un punto de vista concreto y aplicable a América Latina, Varela conceptualiza a la innovación como "el proceso mediante el cual prototipos, modelos, conceptos o ideas se integran al mercado y se ofrecen como bienes para ser adquiridos por los clientes. Es convertir una idea en una empresa" (2008, 263). Agrega que la innovación "exige un conocimiento del mercado, una mentalidad práctica y una orientación empresarial que permita llevar a cabo, en la realidad, la empresa diseñada" (265). Estas definiciones son el eje del presente estudio.

Por lo indicado y para que las organizaciones generen ideas creativas e innovaciones útiles para su entorno socioambiental, tal como lo expresa Bouwen (2001), sus líderes deben entender que el desarrollo, el intercam- 
bio y la integración del conocimiento son esencialmente actividades relacionales. Cuando las personas interactúan, usan un lenguaje común que los delimita para transmitir contenido con base en objetos entendidos recíprocamente; al mismo tiempo, experimentan y actúan de modo relacional. En las empresas, se debe propender que estas prácticas relacionales sean integradas y explícitas en grupos de trabajo multidisciplinarios, en los que unos pueden aportar más contenido y otros, más trabajo, de forma equilibrada, respetando las opiniones de todos y sin abusos de poder ni mínimo esfuerzo por parte de los jefes. Hay que mantener siempre presente que "el cambio o la innovación es una redefinición del problema en esencia" (Bouwen 2001, 368).

Agrega que estas prácticas relacionales, en cualquier espacio interactivo o de intercambio de entre por lo menos dos actores, tienen consecuencias en las características de la relación y lleva a algunas metas en común, aunque no siempre orientadas al beneficio de la organización. En ellas normalmente se redefine el contenido del mensaje y, por ende, la relación; por este motivo, estos enfoques deben ser tomados en cuenta. Concluye diciendo que:

En nuestro turbulento mundo intensivo en conocimiento tenemos que inventar nuevas prácticas relacionales donde se fomente la producción de contenido y se estimulen las relaciones [...]. Hay un desafío por delante para científicos y profesionales sociales para crear una agenda, un entorno físico, un grupo de personas, alguna actividad conjunta, mucha improvisación y espíritu social, en resumen, una práctica relacional para cada momento particular donde se requiera sacar adelante un problema $\mathrm{y}$, al mismo tiempo, sentirse miembro de una comunidad de práctica. (Bouwen 2001, 368)

Por otro lado, dichas prácticas relacionales deben ser dialógicas y no monológicas, como plantea Shotter: "Una actividad polifónica, con uno o varios centros, márgenes, constituyente de una tradición de argumentación que suministra una serie de posturas a toda una pluralidad de conciencias, cada una con su propio mundo" $(2001,98)$. Este enfoque es muy importante para el construccionismo social, ya que presenta una versión dialógica conversacional llamada retórico-respondiente; esto debido a que el habla no solo representa, sino que es reactiva y usa metáforas para que quien escucha establezca sus propias relaciones mentales con base en los enunciados del que habla. Dicha corriente considera que "el flujo y la actividad son lo primario y juzga problemática la conquista de la estabilidad" (Shotter 2001, 265), al contrario que otras ciencias sociales. 
Además, estos entornos deben aplicar el concepto y ser similares a las comunidades de práctica de Wenger (2001), que son omnipresentes a lo largo $\mathrm{y}$ ancho de nuestras vidas y en las que una persona puede pertenecer a varias, de manera intermitente. Normalmente, estas comunidades realizan alguna actividad común que le da sentido, por lo que desarrollan significados de lo que hacen. Además, crean y recrean una identidad grupal; se aprende mucho en estas comunidades de práctica, tal vez más que en aulas, porque son muy variadas y, por lo general, existe una motivación para pertenecer a ellas. Por ejemplo, al joven apasionado por la música le gusta más estar ensayando con su banda que en clases de Música en el colegio. En resumen, las comunidades de práctica tienen "al mismo tiempo el carácter sorprendente de la novedad y la familiaridad olvidada de lo evidente, aunque quizá sea esta la marca de nuestras instituciones más útiles" (Wenger 2001, 25).

Como resultado de este proceso, se pueden generar ciertas competencias de innovación, como las determinadas por Dyer, Gregersen y Christensen (2012), quienes luego de estudiar a innovadores disruptivos y a sus empresas (destrucción creativa schumpeteriana), determinaron que las principales competencias de innovación, asociadas con su capacidad de descubrimiento y que se pueden desarrollar en empleados, estudiantes y a nivel personal, son las siguientes.

a) Asociación: es la habilidad de relacionar lo que aparentemente no se relaciona y hacer conexiones inesperadas entre distintas áreas de conocimiento, sectores industriales e incluso regiones o países.

b) Cuestionamiento: es el catalizador creativo para las siguientes competencias. Representa la capacidad de hacer cientos de preguntas que motivan la reflexión, para entender mejor lo que es y lo que podría ser cualquier cosa. Suelen ser preguntas disparatadas que desafían lo establecido y cuestionan el poder con intensidad y una frecuencia no habitual.

c) Observación: se trata de un examen intenso y atento del mundo que los rodea y su funcionamiento, para luego descubrir las cosas que no funcionan y, así, crear conexiones entre información que inicialmente parecería inconexa, pero que puede generar ideas inusuales.

d) Creación de redes (o networking): implica pensar de forma creativa y conectar las ideas de nuestra área de conocimiento con las personas de otros campos e, inclusive, con quienes están fuera de nuestra área de 
influencia. De esta forma, es posible adquirir perspectivas radicalmente distintas al encontrar y probar ideas dentro de redes formadas por una amplia variedad de individuos.

e) Experimentación: aunque las competencias anteriores generan información sobre lo que había (el pasado) y lo que hay (el presente), la experimentación es el método más viable para generar datos sobre lo que podría funcionar más adelante (el futuro).

\section{E-learning en entornos virtuales de aprendizaje}

El e-learning es una herramienta de apoyo a los métodos más actuales de enseñanza-aprendizaje centrados en el estudiante, ya sea presencial, a distancia o de manera combinada-comunidades de conocimiento y aulas inteligentes (Tissenbaum y Slotta 2012), y ecosistemas de aprendizaje digital o learning management systems (LMS) (Ellis 2009; Szabo 2002)- con elementos que permiten aprovechar las nuevas tecnologías, como los planteados por el Reporte Horizon para la Educación Superior (Johnson et al. 2014). Su uso se ha difundido a través del mundo con sistemas de e-learning como Moodle, WebCT y Blackboard, con varios casos de éxito.

Un verdadero massive open online course (MOOC) -en español, curso online masivo abierto (COMA) - es diferente de un cursillo online que podemos seguir, por ejemplo, revisando videos en YouTube. Dicha diferencia se fundamenta en la existencia de un trabajo interdisciplinario dirigido a participantes globales de diferentes niveles, que reciben atención más personalizada. Además, se debe contar con un sistema de evaluación que puede incluir autoevaluación y evaluación de sus pares o compañeros de curso (Marauri 2014).

La diferente ubicuidad y temporalidad del e-learning, con respecto a la educación presencial, son presentadas por Bernárdez (2007). Él manifiesta que en la educación virtual puede existir una formación presencial y simultánea (sincrónica), pero es muy común la formación a distancia y con diferentes tiempos (asincrónica). Sin embargo, a pesar de estas barreras, los EVA deben lograr este nivel de diálogo para la construcción de conceptos mentales comunes: 
Figura 6

Tipos de entornos virtuales de aprendizaje (EVA)

\begin{tabular}{|c|c|c|}
\hline & Simultáneo & Diferido \\
\hline Mismo lugar & $\begin{array}{l}\text { Formación } \\
\text { presencial }\end{array}$ & $\begin{array}{c}\text { Estudio dirigido } \\
\text { Instrucción programada }\end{array}$ \\
\hline Diferente lugar & $\begin{array}{l}\text { Formación } \\
\text { a distancia } \\
\text { sincrónica }\end{array}$ & $\begin{array}{c}\text { Formación a distancia } \\
\text { asincrónica }\end{array}$ \\
\hline
\end{tabular}

Fuente: Bernárdez $(2007,20)$.

Para relacionar las teorías y los conceptos presentados, se parte de lo indicado por Lau y Tsui (2009), quienes manifiestan que, hasta dicho año, no se habían realizado suficientes estudios serios sobre la sinergia, eficacia funcional e integración de la gestión del conocimiento en EVA. En su investigación, concluyen que una de las principales causas por la que los individuos no alcanzan los objetivos de aprendizaje puede ser una selección equivocada de los métodos ubicuos de transmisión de conocimientos.

Las limitaciones en la integración de la gestión de conocimiento y el e-learning están basadas en una baja implementación en entornos productivos, por carecer de especificaciones técnicas y soporte de aplicaciones. Por este motivo y con base en estudios generados desde las ciencias informáticas y educativas, se recomienda "basar la integración en un terreno común para ambas disciplinas, que sería la educación” (Judrups 2015, 162). Por último, Strunga (2015) agrega que las universidades deben generar modelos de gestión del conocimiento por carreras, basados en el desarrollo de identidades profesionales, pero con apertura a la innovación.

El reto primordial para que lo indicado se cumpla es que vivimos en una época que ha sido llamada "modernidad líquida", concepto que no fue inventado pero sí desarrollado por el pensador polaco Zygmunt Bauman (2005); lo líquido implica la capacidad de las formas de adaptarse y fluir. Este concepto tiene connotaciones positivas, por la capacidad de las nuevas generaciones de adaptarse con mayor rapidez a los cambios. Sin embargo, desde una perspectiva negativa, se puede decir que lo que se ha "licuado" son los bienes y 
servicios - de poca duración y con obsolescencia tecnológica programada-, los procesos y las relaciones humanas -cada vez más fugaces, remotas e impersonales- y hasta los principios y valores, esos componentes que antes eran sólidos e inamovibles y con los que podíamos construir los cimientos de nuestra sociedad. Son tiempos en que los cambios son tan vertiginosos, instantáneos y caóticos, que la novedad diaria es la norma y, por ende, existe una incertidumbre casi completa sobre el presente y el futuro. Esta es una situación que no solo se presenta en los países desarrollados, sino que ha existido una "propagación global de la forma de vida moderna, que ha alcanzado a estas alturas los límites más remotos del planeta. Ha anulado la división entre centro y periferia" (Bauman 2010, 79).

Otros signos de esta vida líquida son la necesidad de gratificación inmediata y la aversión al compromiso y al esfuerzo permanente: una cultura del distanciamiento, de la discontinuidad y del olvido; lo que Bauman llamó "el síndrome de la impaciencia [...]. El tiempo ha llegado a ser un recurso, quizá el último, cuyo gasto se considera unánimemente abominable, injustificable e intolerable" (2008, 21-22). El filósofo agrega que los retos de la educación a los hombres y las mujeres de la modernidad líquida se basan en la perspectiva de que el conocimiento ya no se adquiere en un proceso de aprendizaje durante toda la vida, sino que es un producto perecible y cambiante, de utilidad limitada. Esto, básicamente, por la "naturaleza errática y esencialmente impredecible del cambio contemporáneo" (Bauman 2008, 31), así como por la sobresaturación de información en el mundo.

El e-learning para los millennials de la Generación Y (nacidos aproximadamente entre 1980 y 1999) y los posmillennials de la Generación Z (entre 1997 y 2012), que están formándose en las universidades, debe considerar su naturaleza cambiante y relativamente poco comprometida a esfuerzos de largo plazo. De esta forma, se podrá aprovechar de mejor manera su condición de nativos digitales, sus habilidades con la tecnología y las redes sociales, y su avidez para obtener información en cualquier momento, sobre todo a través de imágenes, audios y videos (Avilés y Eastman 2012). Sin embargo, la estructuración de los cursos e-learning para estos grupos demográficos debe considerar no solo la transmisión de habilidades técnicas para el trabajo, el saber hacer, sino el deseo de saber, de adquirir conocimientos a lo largo de su vida y, sobre todo, la formación valórica de ciudadanos conscientes de 
sus derechos democráticos, que recuperen el espacio público del diálogo, que busquen su propia identidad y que aprecien la postergación del premio obtenido como resultado de un esfuerzo continuado.

Los educadores debemos "aprender el aún más difícil arte de preparar a las próximas generaciones para vivir en semejante mundo" (Bauman 2008, 46). Esta premisa es más apremiante, a la vez que compleja, en América Latina y en países como Ecuador, con indicadores socioeconómicos desfavorables y una importante brecha digital en comparación con otras regiones del mundo. Inclusive dentro de nuestros países, dichas desigualdades se remarcan al analizar las diferencias entre sectores urbanos y rurales. La educación virtual a distancia aparece como una interesante alternativa para superar parte de esa inequidad en el acceso a información y el conocimiento por parte de la población rural.

El desarrollo de sistemas e-learning que consideren las teorías, las perspectivas y los conceptos indicados anteriormente, así la posibilidad de medir su impacto en el desarrollo de sus habilidades de innovación, podría preparar adecuadamente a nuestros profesionales, empleados y trabajadores para la industria $4.0,{ }^{1}$ basada en el auge inicial de las computadoras, la automatización, la robótica y las energías renovables, entre otros.

La industria 4.0 está transformando a fondo a las organizaciones en general, no solo las productivas, ya que, dentro del capitalismo cognitivo, se está expandiendo el trabajo inmaterial hasta llegar al dominio del trabajo intelectual, donde las ideas, el diseño, la publicidad, el mercadeo, etc., tienen más valor que el trabajo físico de transformación. Labores como la fabricación de bienes, limpieza, seguridad, entre otras, corresponden al 47\% de empleos que desaparecerán en las próximas dos décadas, según la Universidad de Oxford, por efectos de la robótica y la automatización; en esta sociedad red, hemos pasado "de la galaxia de Gutenberg a la galaxia de McLuhan" (Castells 2004, 362), considerando la democratización de la educación que logra-

1. Se conoce como primera Revolución Industrial a la mecanización que se consiguió con el movimiento impulsado por el poder del agua y, sobre todo, del vapor. La segunda es la descrita por Harvey en su libro La condición de la posmodernidad (2012), y está caracterizada por el fordismo, la producción en masa y la línea de ensamblaje con máquinas impulsadas por electricidad. La industria 4.0, llamada la cuarta Revolución Industrial, empezó después de 2010 y complementa y amplía la tercera, de inicios del siglo XXI. 
ron la imprenta y los libros hasta la masificación de la información a través del internet en la sociedad de la información, dentro de la aldea global que soñó el gran visionario y comunicador Marshall McLuhan. Aunque hemos presenciado el ascenso de la cultura de los medios de comunicación en masa, Castells no considera que vivamos en esa aldea global, sino en "chalecitos individuales, producidos a escala global y distribuidos localmente" (374).

En este espacio de flujos, donde las localidades se desprenden de sus significados y se reintegran en redes y collages de imágenes y de tiempo atemporal, donde "pasado, presente y futuro pueden reprogramarse para interactuar mutuamente en el mismo mensaje" (408), no es tan cierto que el medio es el mensaje, como profetizaba McLuhan. En el nuevo sistema, el mensaje es el mensaje, por la diversidad potencial de contenidos. Esto lo saben los creadores emprendedores, que tienen "la capacidad de diferenciar un producto que produzca el mayor potencial competitivo" (401). De igual forma, la cultura de la virtualidad real captura a la realidad, la sumerge en un escenario de imágenes virtuales, en un mundo de make-believe (hacer creer), donde "las apariencias no están solo en las pantallas a través de las cuales se comunica la experiencia, sino que se convierten en la experiencia” (406).

\section{Discusión y conclusiones}

\section{Análisis de escenarios de educación virtual \\ para el desarrollo de competencias de innovación basadas en estilos de aprendizaje personal}

Beckman y Barry (2007), con base en el modelo del aprendizaje experimental de Kolb (2014), plantean que el conocimiento pleno surge de la combinación de dos componentes: uno analítico, en el reino de la teoría, que, usando principios, medidas y paradigmas de investigación, lleguen de un propósito a un descubrimiento; y un componente sintético en el reino de la práctica, que también usa medidas y principios, pero orientados hacia paradigmas de aplicación, con el fin de trabajar en una invención.

Además, manifiestan que la innovación es un proceso de aprendizaje que puede ser enseñado y medido con el fin de determinar la predisposición de 
los individuos a innovar y el desarrollo de sus competencias de innovación, basándose en sus respectivos estilos de aprendizaje: asimilación, divergencia, convergencia y conveniencia.

Figura 7

El proceso de innovación y los estilos de aprendizaje

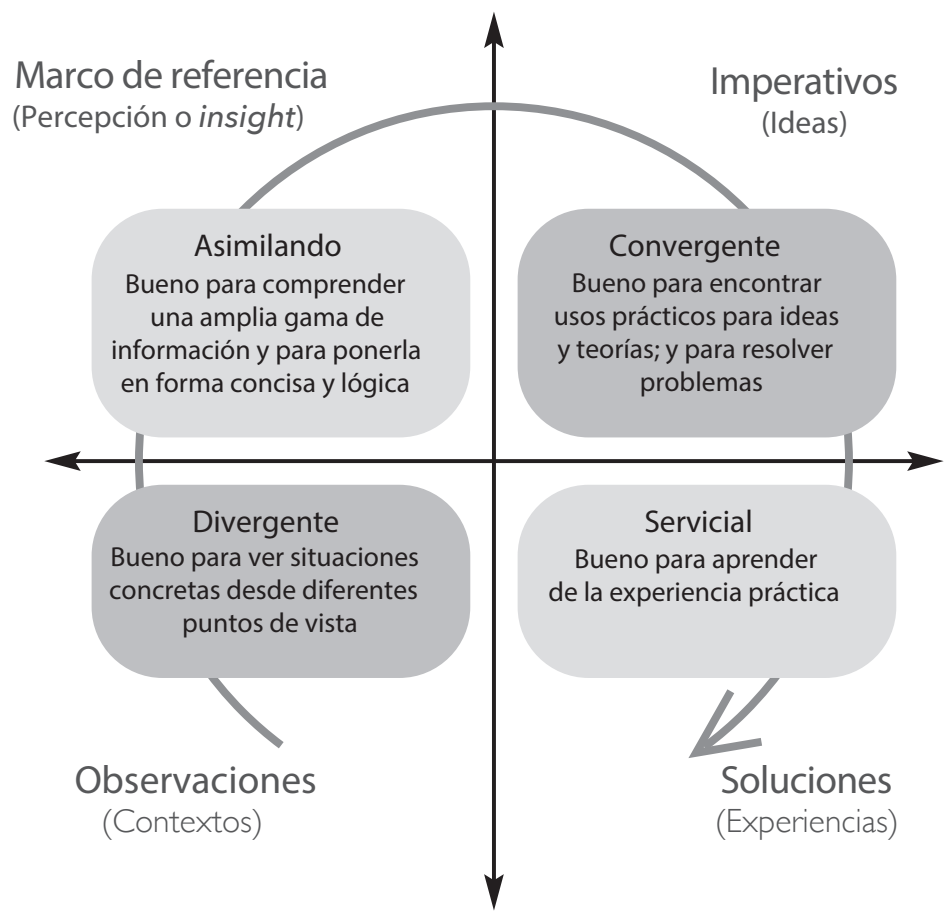

Fuente: Beckman y Barry $(2007,47)$.

\section{Escenario 1: individuos con estilo de aprendizaje divergente}

Son personas con tendencia a aprender luego de una observación reflexiva de experiencias concretas, lo que les permite analizar las situaciones específicas desde múltiples puntos de vista. Van a ser los artistas, quienes, dentro del contexto, observarán un aspecto que valga la pena ser resaltado o 
encontrarán un problema puntual. "Haciendo un paralelismo con las fases de una historia, serán quienes harán un llamado a la aventura" (Beckman y Barry 2007, 45), el érase una vez, que nos narra la situación inicial de nuestros héroes o heroínas.

Los EVA para el e-learning orientado a este tipo de individuos deben presentar casos de estudio - puede ser en texto, pero preferiblemente en video y relacionados con innovación-, por ejemplo, de nuevos productos, en los que no se presente la solución tomada. Esto, con el fin de que los participantes puedan reflexionar sobre el caso y ponerlo en el contexto apropiado. Además, se deben configurar chats donde los participantes puedan exponer sus diferentes puntos de vista al problema para llamar la atención del resto y aportar con posibles cursos de acción. El uso de imágenes, figuras, gráficos, mapas mentales, etc., debe ser profuso, por las características artísticas de estos individuos. A nivel de actividades prácticas, se les puede enviar, para luego evaluarlas, investigaciones etnográficas en las que puedan realizar observaciones participativas y no participativas, entrevistas formales e informales, diarios de campo, sondeos en la web, etc.

\section{Escenario 2: individuos con estilo de aprendizaje de asimilación}

Son quienes aprenden observando y analizando conceptos abstractos, lo que les permite entender una gran cantidad de datos e información. Tienen la capacidad de sintetizarla para ponerla de forma lógica y concisa en beneficio de los demás. Con similitud a los escritores, podrán establecer marcos conceptuales de referencia de los que emergen vacíos -entendidos como oportunidades no descubiertas-y percepciones o insights -esos espacios entre lo que es y podría ser a nivel de uso, usabilidad, sentido, etc.- - Todo esto, con el objetivo de contestar la gran pregunta, ¿qué es lo que falta?, para seleccionar una parte más específica del problema y, como en un cuento, presentar el punto de quiebre que rompa la situación habitual y a partir del cual para nuestros héroes o heroínas "el gran viaje comienza" (Beckman y Barry 2007, 45).

Los EVA para el e-learning orientado a este tipo de individuos deben, de igual forma, presentar casos de estudio, pero proveyendo una mayor cantidad de datos, tanto conceptuales como numéricos, inclusive aquellos no relevantes para el caso, con el fin de ejercitar su capacidad de síntesis y se- 
lección de información pertinente. También se deben configurar chats donde los participantes puedan presentar, de forma lógica y resumida, ese importante espacio vacío que otros no han percibido y sobre el cual es posible actuar. Como tareas, se puede solicitar la elaboración de diagramas AEIOU (actividades, ambiente, interacciones, objetos y usuarios, por sus siglas en inglés), la identificación de historias basadas en necesidades emocionales o no emocionales, matrices de comportamiento y hábitos de consumo, líneas temporales, mapas de proceso, representaciones fotográficas, etc.

\section{Escenario 3: individuos con estilo de aprendizaje convergente}

Estos participantes aprenden experimentando con base en una conceptualización abstracta previa. De esta forma, es muy posible que estos voceros encuentren usos prácticos a ideas y teorías presentadas por los escritores. Generarán "propuestas de valor imperativas que debe cumplir el nuevo concepto" (Beckman y Barry 2007, 41). Luego del intermedio que narra el nudo de la historia, nuestros héroes o heroínas realizarán una valerosa hazaña que los enrumbará al final del cuento.

Los EVA para el e-learning orientado a este tipo de individuos deben usar los casos de estudio, pero en este caso podrían tener la solución tomada o el producto generado, para que los participantes analicen los aspectos positivos o negativos de esa decisión. La utilización de chats para contacto permanente entre participantes y profesor permitirá validar dichas propuestas de valor entre todos y empezar a vislumbrar una posible solución final o el diseño definitivo del producto. La evaluación se puede realizar a partir de tareas grupales o individuales en las que tengan que realizar una descripción de los beneficios tangibles para el consumidor, la definición de los resultados más importantes alcanzados en las posibles soluciones al problema, la identificación de nuevas necesidades del consumidor, la definición de los principios de diseño del producto, etc.

\section{Escenario 4: individuos con estilo de aprendizaje complaciente}

Los individuos cuyo aprendizaje se fundamenta en experiencias concretas, propias o de terceros, que los llevan a experimentar activamente para 
acumular nuevas experiencias prácticas, se acomodan perfectamente dentro de la expresión aprender haciendo. Serán líderes proactivos que querrán construir con sus propias manos el futuro al que aspiran. Serán quienes seleccionarán la solución que crean correcta, aun a riesgo de estar equivocados. Sus acciones traerán enseñanzas para otros, así como los aciertos y errores de los demás les enseñarán nuevas moralejas.

Los EVA para el e-learning orientado a este tipo de individuos deben usar los casos de estudio grupales, en los que seguramente tendrán el voto dirimente para presentar el resultado final. Los chats $u$ otras formas de contacto, tanto sincrónicas como asincrónicas, les permitirán liderar y hacer seguimiento de todo el proceso de toma de decisiones y elaboración del informe de resultados. Los trabajos prácticos pueden estar relacionados con la generación de conceptos de producto, la elaboración de prototipos, el análisis morfológico de productos, la definición de criterios de selección versus la competencia, la realización de pruebas de concepto (por ejemplo, ferias de ideas creativas, ruedas de negocios, etc.), entre otros.

\section{Conclusiones}

Desde hace muchos años se sabe que una formación de calidad es aquella que se ajusta de la mejor manera a los antecedentes, las formas, los estilos y las expectativas de todos y cada uno de los futuros profesionales en las universidades, así como del personal a ser capacitado dentro de las empresas. Esta afirmación es válida en la modalidad presencial, a pesar de las dificultades que generan los grupos numerosos, las limitaciones de tiempo y los programas académicos estrictos. Esa necesidad de adaptación también es necesaria en el e-learning, a pesar que existen otras complicaciones, como una cantidad mayor de participantes - en cursos masivos en línea-y la separación del profesor o tutor en espacio y tiempo.

Teniendo en cuenta las limitaciones en tiempo, distancia y acceso a la educación presencial que tiene un alto porcentaje de la población -bachilleres, adultos mayores o con capacidades especiales, personas pertenecientes a ciertas etnias, trabajadores con horarios especiales, etc.--, esta podría ser una alternativa viable de oferta académica para que puedan obtener un título 
de tercer o cuarto nivel en una universidad ecuatoriana que realmente los prepare para los retos del mundo globalizado, en un país donde se requiere impulsar la innovación. De igual forma, en las organizaciones con o sin fines de lucro resulta cada vez más complicado movilizar y reunir a los colaboradores, que laboran en horarios diferentes y oficinas distantes. Por esta razón, también es válida la implementación de programas de capacitación, formación y/o desarrollo basados en e-learning, que aprovechan las tecnologías actuales de información y comunicación.

Las teorías de gestión del conocimiento resaltan al individuo como la base de la creación de dicho conocimiento; se enfocan en cómo aprende según su experiencia previa, qué hace, qué lo relaciona con su entorno comunitario y qué lo vuelve la mejor versión de sí mismo. Esos saberes y formas diferentes de aprender pueden aportar decididamente a la generación de innovación, al considerar al estudiante y a los otros participantes del proceso educativo. Las universidades ecuatorianas, en conjunción con las organizaciones del sector productivo privado y el Estado, en ese triángulo de la innovación planteada por Sábato y Botana (1978), deben conformar circuitos internacionales para generación de conocimiento, con organizaciones reticulares de trabajo que luchen contra una exclusiva apropiación privada del trabajo y del propio conocimiento.

Por ejemplo, la investigación de Ramírez-Anormaliza (2017) para su tesis doctoral demuestra que existe una aceptación de los sistemas e-learning en universidades, empresas y otro tipo de organizaciones. Sin embargo, su implementación como apoyo a la educación presencial o semipresencial aún es incipiente, así como lo son las investigaciones que buscan establecer los efectos de esta modalidad de educación en el desarrollo de competencias de innovación y en el desempeño académico o laboral.

De igual forma, se espera que dicha innovación no solo se centre en productos -sobre todo tecnológicos- que, por efecto de la comunicación publicitaria, presionen a los consumidores a comprarlos por obsolescencia ficticia basada en modas o aspiraciones. Esta situación afecta la economía personal y familiar de los individuos, además de la sostenibilidad ambiental de nuestro planeta. El reto es innovar personas, procesos, ambientes y conocimientos que generen un mejor futuro para la sociedad en su conjunto. 
Figura 8

\section{El triángulo de la innovación}

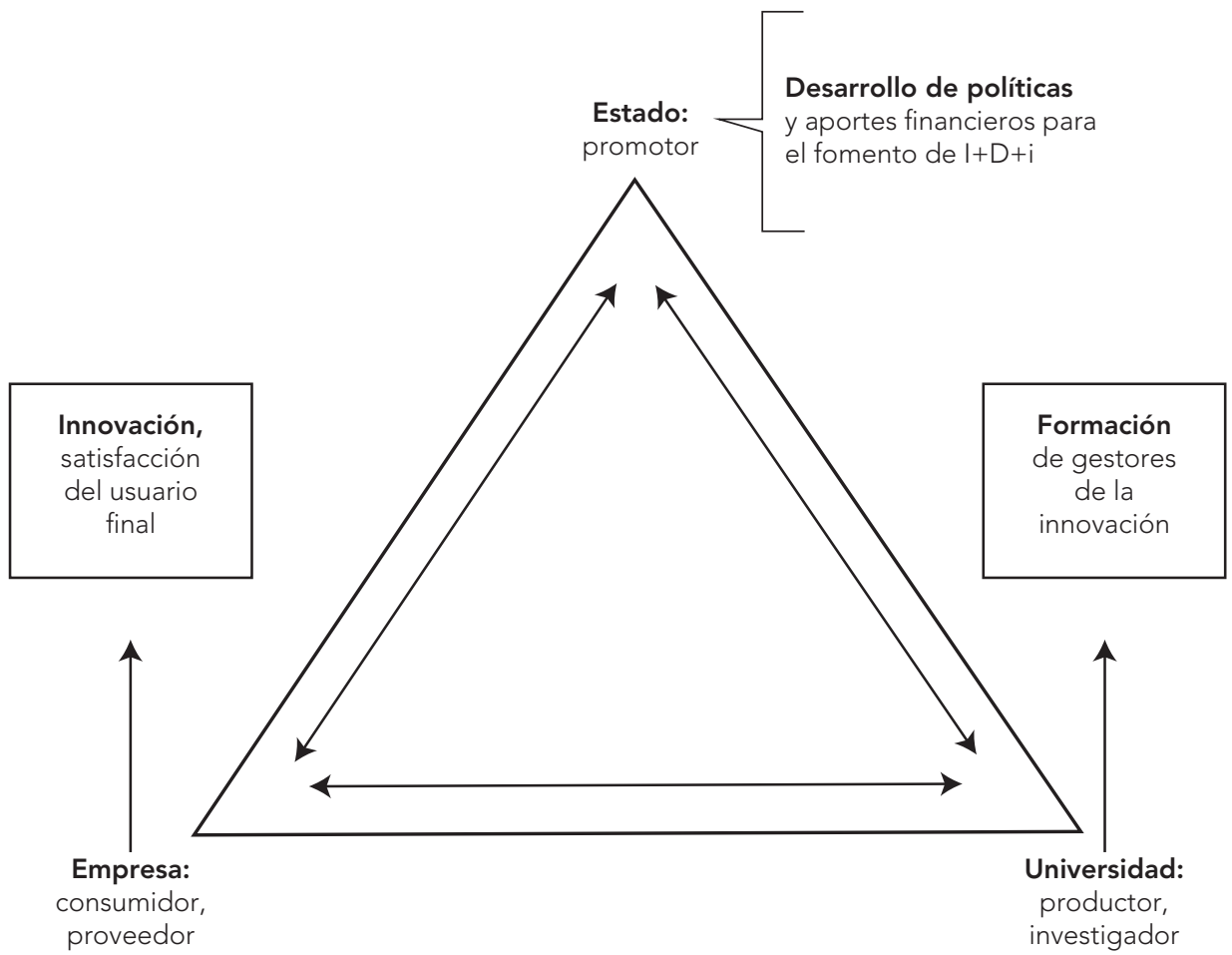

Fuente: Sábato y Botana $(1978,7)$.

Como conclusión final, se debe considerar que tanto los profesores y capacitadores como los jefes tipo coach deben identificar los estilos de aprendizaje de las personas a su cargo. De esa forma, sus EVA considerarán y estarán alineados de la mejor forma posible con su forma de aprender. Esto facilitará el desarrollo de competencias de innovación, como la predisposición a observar, cuestionar y experimentar, así como a asociarse y formar redes.

En el proceso de conformación de grupos de estudio o de trabajo, se debe propender a la integración y combinación de individuos con diferentes estilos. Así se logrará formar equipos cuyos integrantes aporten con sus ob- 
servaciones y acceso a la información teórica y, también, generen soluciones concretas y estén dispuestos a llevarlas a la práctica. Los diferentes criterios enriquecen la discusión y permiten completar adecuadamente el proceso que genera la destrucción creadora de la innovación.

\section{Referencias}

Arrow, Kenneth J. 1962. "The Economic Implications of Learning by Doing". The Review of Economic Studies 29 (3): 155-173. https://doi.org/10.2307/2295952

Avilés, María, y Jacqueline K. Eastman. 2012. "Utilizing Technology Effectively to Improve Millennials' Educational Performance". Journal of International Education in Business 5 (2): 96-113. https://doi.org/10.1108/18363261211281726

Barberà, Elena. 2008. Aprender e-learning. Barcelona: Planeta.

Bauman, Zygmunt. 2005. Modernidad líquida. Buenos Aires: Fondo de Cultura Económica.

---. 2008. Los retos de la educación en la modernidad líquida. Barcelona: Gedisa.

---. 2010. Tiempos líquidos: vivir en una época de incertidumbre. Barcelona: Tusquets.

Beckman, Sara L., y Michael Barry. 2007. "Innovation as a Learning Process: Embedding Design Thinking”. California Management Review 50 (1): 25-56. https://doi. org/10.2307/41166415

Bernárdez, Mariano L. 2007. Diseño, producción e implementación de e-learning: metodología, herramientas y modelos. Bloomington, AuthorHouse.

Bjornali, Ekaterina S., y Liv Anne Støren. 2012. "Examining Competence Factors that Encourage Innovative Behaviour by European Higher Education Graduate Professionals". Journal of Small Business and Enterprise Development 19 (3): 402-423. https://doi. org/10.1108/14626001211250135

Bouwen, Rene. 2001. "Developing Relational Practices for Knowledge Intensive Organizational Contexts". Career Development International 6 (7): 361-369. https://doi.org/10.1108/ EUM0000000006057

Brito Albuja, José Guillermo. 2014. Aprehendizaje y desarrollo en la educación infantil. Quito: Universidad San Francisco de Quito.

Castells, Manuel. 2004. "La Sociedad red". En La era de la información: economía, sociedad y cultura. Vol. I: 359-408. Buenos Aires: Siglo XXI Editores Argentina S.A.

Davenport, Thomas H., y Laurence Prusak. 2001. Conocimiento en acción: cómo las organizaciones manejan lo que saben. Buenos Aires: Pearson Education.

Daza, Carmen. 2012. "Modelo de efectividad de la red de innovación tecnológica. Una visión desde el constructivismo social". Tesis doctoral, Universidad Andina Simón Bolívar, Sede Ecuador, Quito. 
Drucker, Peter. 1993. La sociedad postcapitalista. Bogotá: Norma.

Dyer, Jeff, Hal Gregersen y Clayton M. Christensen. 2012. El ADN del innovador: claves para dominar las cinco habilidades que necesitan los innovadores. Barcelona: Planeta.

Ellis, Ryann K. 2009. "A Field Guide to Learning Management Systems". American Society for Training \& Development. Learning Circuits 8 (5): 1-7. http://www.ncbi.nlm.nih.gov/ pubmed/20616636

Harvey, David. 2012. La condición de la posmodernidad: investigación sobre los orígenes del cambio cultural. Buenos Aires: Amorrortu.

Johnson, Bjorn. 1992. "Institutional Learning". En National Innovation Systems: Toward a Theory of Innovation and Interactive Learning, editado por Bengt-Ake Lundvall, 23-45. Londres: Pinter Publishers.

Johnson, Larry, Samuel Adams Becker, Victoria Estrada, Alex Freeman, Panagiotis Kampylis, Riina Vuorikari e Yves Punie. 2014. NMC Horizon Report: 2014 Higher Education Edition. Austin, The New Media Consortium.

Judrups, Janis. 2015. “Analysis of Knowledge Management and E-Learning Integration Models”. Procedia Computer Science 43 (0): 154-162. https://doi.org/10.1016/j.procs.2014.12.021

Kolb, David A. 2014. Experiential Learning: Experience as the Source of Learning and Development. Londres: Pearson Education.

Lane, Peter, y Michael Lubatkin. 1998. "Relative and Capacity Learning". Strategic Management Journal 19 (5): 461-477. https://doi.org/10.1002/(SICI)1097-0266(199805)19:5\%3 C461::AID-SMJ953\% 3E3.0.CO;2-L

Lau, Adela, y Eric Tsui. 2009. "Knowledge Management Perspective on E-Learning Effectiveness". Knowledge-Based Systems 22 (4): 324-325. https://doi.org/10.1016/j. knosys.2009.02.014

Lundvall, Bengt-Ake. 1992. National Systems of Innovation. Towards a Theory of Innovation and Interactive Learning. Londres: Pinter Publishers.

---. 1999. "La base del conocimiento y su producción”. Ekonomiaz (45): 14-37. https://dialnet. unirioja.es/servlet/articulo? codigo $=265836$

---, y Bjorn Johnson. 1994. "The Learning Economy". Journal of Industry Studies 1 (2): 2342. https://doi.org/10.1177/003172171009100619

Marauri, Pedro María. 2014. "Figura de los facilitadores en los cursos online masivos y abiertos (COMA/MOOC): nuevo rol profesional para los entornos educativos en abierto". Revista Iberoamericana de Educación a Distancia 17 (1): 18-25. http://revistas.uned.es/ index.php/ried/article/view/11573

Nonaka, Ikujiro, y Hirotaka Takeuchi. 1999. La organización creadora del conocimiento. Ciudad de México: Oxford University Press.

---, y Ryoko Toyama. 2006. "Creating Sustainable Competitive Advantage through Knowledge-Based Management". Office of the Public Sector Development Commision OPDC, 1-70. https://www2.opdc.go.th/uploads/files/nonaka.pdf

OCDE. 2007. Manual de Oslo. Vol. 30. Oslo: Eurostat. 
Ramírez-Anormaliza, Richard. 2017. "Modelo de aceptación de los sistemas e-learning en las universidades: un enfoque del modelo de aceptación de la tecnología (TAM) ajustado al Ecuador". Tesis doctoral, Universitat Politècnica de Catalunya, Barcelona. http://hdl. handle.net/10803/405799\%0A

Ramos, Valentina. 2017. "Dimensión humana del espacio organizacional. Cap. 1: Los procesos humanos que dinamizan el espacio organizacional. El aprendizaje organizacional y la gestión del conocimiento", 6-18. Quito: Escuela Politécnica Nacional.

Rodríguez Gómez. 2006. "Modelos para la creación y gestión del conocimiento: una aproximación teórica". Educar 37 (5): 25-39. https://www.redalyc.org/pdf/3421/342130826003.pdf

Sábato, Jorge, y Natalio Botana. 1978. "La ciencia y la tecnología en el desarrollo futuro de América Latina". Revista de Integración 3 (2): 4-11. https://go.aws/2XkIc61

Schumpeter, Joseph A. 1997. Teoría del desenvolvimiento económico: una investigación sobre ganancias, capital, crédito, interés y ciclo económico. Ciudad de México: Fondo de Cultura Económica.

Shotter, John. 2001. Realidades conversacionales. Buenos Aires: Amorrortu.

Strunga, Alexandru. 2015. "The Integration of Virtual Learning Communities into Universities' Knowledge Management Models". Procedia-Social and Behavioral Sciences 197 (8): 2430-2434. https://doi.org/10.1016/j.sbspro.2015.07.306

Szabo, Michael. 2002. "CMI Theory and Practice Historical Roots of Learning Management". World Conference on E-Learning in Corporate, Government, Healthcare, and Higher Education, 929-936. https://www.learntechlib.org/p/15322

Tissenbaum, Lui, y James Slotta. 2012. "Co-Designing Collaborative Smart Classroom Curriculum for Secondary School Science". Journal of Universal Computer Science 18 (3): 327-352. https://doi.org/10.3217/jucs-018-03-0327

Varela, Rodrigo. 2008. Innovación empresarial: arte y ciencia en la creación de empresas. Bogotá: Pearson.

Velayudhan, Mily, y K. Maran. 2010. "A Study on Competencies of the Employees of 21 St.”. Management Prudence 1 (1): 28. https://search.proquest.com/docview/1478031814? pq-origsite=gscholar

Wenger, Etienne. 2001. "Introducción: una teoría social del aprendizaje". En Comunidades de práctica: aprendizaje, significado e identidad, editado por Centro Nacional de Artes, 19-36. Barcelona: Paidós. 